\title{
Influence of infertility diagnosis on pregnancy outcome in ART
}

\author{
Erin E. Eppsteiner, MD, ${ }^{1}$ Mark K. Santillan, MD, ${ }^{1}$ Barbara J. Stegmann, MD, MPH ${ }^{1}$
}

Keywords: IVF outcome, gestational age, birth weight, single embryo transfer

\begin{abstract}
Objective: To determine the influence of the infertility diagnosis on gestational age (GA) and birth weight $(B W)$ of children conceived using assisted reproductive technology (ART).
\end{abstract}

Design: Retrospective cohort.

Setting: University-affiliated infertility clinic.

Patients: Women with a singleton live birth following their first fresh ART cycle with autologous oocytes.

Interventions: Patients were stratified into groups based on infertility diagnosis. GA and $B W$ of their infants were compared.

Main Outcome Measures: $G A$ and BW of children conceived using ART.

Results: 397 women were included. Average $G A$ in the cohort was $38.7 \pm 2.3$ weeks and average BW was $3301.5 \pm 633.8$ grams. Maternal age, BMI, and parity were significantly different between groups. After controlling for these factors and stratifying by infertility diagnosis, there was no difference in GA or BW in infants conceived with ART.

Conclusions: Contrary to previously reported data, there was no difference in GA or BW in infants conceived with ART when stratified by infertility diagnosis. Our results were not different from the national population; however GA was one week longer in our cohort than in the national ART database. These data suggest a difference exists between our cohort and the ART population at large. We propose that the main difference is our institution's focus on single embryo transfer.

${ }^{1}$ Department of Obstetrics and Gynecology, Roy J. and Lucille A. Carver College of Medicine, University of lowa, lowa City, lowa.

\section{Introduction}

In 2009, one out of every sixty-eight babies, or 60,190 infants, born in the United States were conceived using assisted reproductive technologies (ART). ${ }^{1}$ Based on the findings of several previous studies, children conceived using ART have been reported to be at increased risk for preterm birth (PTB) and low birth weight (LBW). In a systematic review by McDonald et al, the odds ratio of PTB was as high as 1.93 and the risk for small for gestational age (SGA) was found to be

Please cite this paper as: Eppsteiner EE, Santillan MK, Stegmann BJ. Influence of infertility diagnosis on pregnancy outcome in ART. Proc Obstet Gynecol. 2014;4(1): Article 1 [ 10 p.]. Available from: http://ir.uiowa.edu/pog/. Free full text article.

Corresponding author: Erin E Eppsteiner, MD, University of lowa, Department of Obstetrics and Gynecology, 200 Hawkins Drive, Iowa City, IA 52242, erin-eppsteiner@uiowa.edu.

Financial Disclosure: Dr. Eppsteiner and Dr. Santillan report no conflict of interest. Dr. Stegmann is an employee of Merck Sharpe and Dohme. However, the work presented here was completed while an employee of the University of lowa.

Copyright: (c) 2014 Eppsteiner et al. This is an open-access article distributed under the terms of the Creative Commons Attribution License, which permits unrestricted use, distribution, and reproduction in any medium, provided the original author and source are credited. 
1.59 for singleton pregnancies achieved with ART compared with singletons that were conceived spontaneously. ${ }^{2}$ Similarly, a 2004 meta-analysis by Jackson et al. examined 12,283 IVF pregnancies and reported an odds ratio of 2.0 for PTB and 1.8 for LBW. ${ }^{3}$ An additional study in 2011 compared sibling pairs who were conceived consecutively; the first was conceived using IVF-ICSI while the second pregnancy was naturally conceived. The authors reported that children conceived with IVF-ICSI had a lower mean birth weight and gestational age at delivery when compared to their naturally conceived siblings. ${ }^{4}$

Although the etiology of these differences is not known, several possible mechanisms have been considered, including advanced maternal age, adverse effects of medications used for ovulation induction, handling and processing of embryos prior to transfer, and the underlying pathology of infertility. Our specific interest lies in the latter. Current data is limited and somewhat contradictory regarding the impact of infertility diagnosis on obstetrical outcome. ${ }^{5,6}$ We hypothesize that infertility diagnosis does in fact have an effect on the obstetrical outcome of singleton pregnancies conceived with ART. Given the lack of consistency in the current literature, we aimed to further evaluate this subject using patients from a single academic medical center.

\section{Materials and Methods}

In this retrospective cohort study, we examined the differences in gestational age at delivery (GA) and birth weight
(BW) by infertility diagnosis in women who underwent singleton birth following their first fresh ART cycle. After receiving IRB approval, information was collected from our internal database on all fresh ART cycles from January 2003 to December 2008. Patients were excluded if they used donor oocytes, frozen embryos, experienced pregnancy loss prior to 20 weeks, or had ongoing multifetal gestation (Figure 1). Prior to oocyte retrieval, patients were treated with either a long luteal GnRH agonist, micro-dose flare agonist, or $\mathrm{GnRH}$ antagonist based on patient characteristics and clinician judgment.

The exposure of interest for this study was the infertility diagnosis. In this cohort, infertility diagnosis was classified as male factor, ovulatory dysfunction, endometriosis, tubal factor, unexplained, or a combination of any of the above diagnoses. Male factor was used as the referent group. Clinically significant covariates analyzed were maternal age at the time of stimulation, race, body mass index (BMI), parity, previous obstetrical outcomes, smoking status, day of embryo transfer, and mode of delivery. The outcomes of interest were GA and BW.

Bivariate and multivariate analyses were performed as appropriate. Linear regression models were developed for each outcome, and stepwise, backward regression was used to test the contribution of each variable to the overall result. Variables remained in the model if estimates were changed by more than $10 \%$. Separate models were developed for both outcomes of interest: GA and BW. We also calculated the mean GA as well as BW for the entire cohort and compared our results with 
those of the composite results from the Society for Assisted Reproductive Technology (SART) database using a Z- score. $^{5}$ Statistical significance was defined as a $p$ value of $<0.05$ using a two-tailed test.

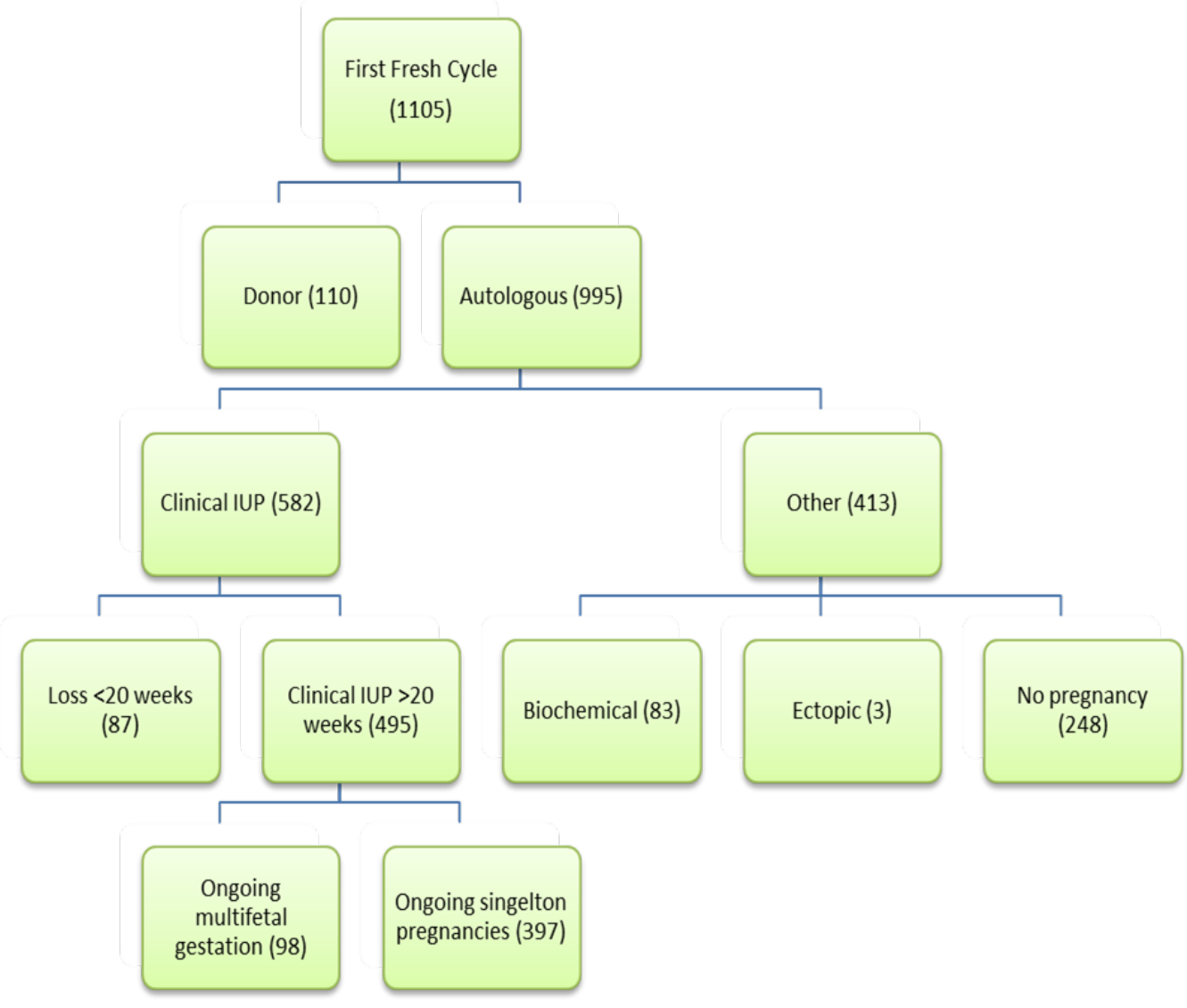

\section{Figure 1. Selection of Study Cohort}

\section{Results}

A total of 1105 women underwent firsttime fresh ART cycles at the University of lowa during the study period. One hundred and ten women used donor embryos and were excluded. Of the remaining 995 patients, 582 cycles resulted in clinical intrauterine pregnancy. Ninety-eight of these were excluded because of multifetal gestation, leaving 397 women who met inclusion criteria for our study (Figure 1).

Infertility diagnosis and ART outcome
Demographic characteristics of the cohort are summarized in Table 1. Average age at oocyte retrieval was 31.8 years $(\mathrm{SD} \pm 4.3)$. Average $\mathrm{BMI}$ was 27.3 (SD \pm 2.4$)$. Race data was only available on 314 women, with a distribution of $92.0 \%$ Caucasian, 3.8\% Asian, 1.3\% African American, 1.3\% Hispanic, and $1.6 \%$ other. Two hundred ninety seven women $(74.8 \%)$ were nulliparous. Two hundred and forty seven women (62.2\%) had day 5 
embryo transfers while 150 (37.8\%) had transfers on day 3 . Of the 397 deliveries, $37(9.3 \%)$ began as a multiple gestation and self-reduced to a singleton. Overall, 254 (64\%) infants were delivered vaginally and $143(36 \%)$ were delivered via cesarean section.

Maternal age, BMI and parity were significantly different between women with different infertility diagnoses $(p<0.05$ for all). Average maternal age (in years), BMI ( $\mathrm{kg} / \mathrm{m} 2)$, and percentage of women with previous delivery are reported in Table 1. Day of embryo transfer and mode of delivery data were not significantly different between the groups. Smoking status data was incomplete with only 84 women reporting a history of smoking. This variable was not included in the final model due to the amount of missing data. Maternal race and previous obstetrical outcome were also incomplete were not included in the model.

Age, BMI, and parity were all found to be significant confounders and were included in the final model. After controlling for these covariates, infertility diagnosis did not impact GA $(p=0.55)$ (Table 2). The second model was then used to evaluate the impact of infertility diagnosis on BW. BW was also not found be to statistically different between women in each diagnostic category $(p=0.61)$ (Table 2). Day of embryo transfer and mode of delivery data did not contribute to the estimates and were dropped from the final model. Post hoc power analysis indicated that we had sufficient power to detect a difference of 461 grams as well as a difference of 2 weeks of average gestational age with an alpha of 0.05 and a power of $80 \%$ when using this dataset.

The overall average GA in our entire cohort was 38.7 weeks ( $\mathrm{SD} \pm 2.3$ ) with a BW of $3301.5 \mathrm{gm}(S D \pm 633.8)$. These did not differ from CDC Birth Data in 2009 for GA and BW which were 38.7 weeks $(\mathrm{SD} \pm 2.4)$ and $3296 \mathrm{gm}$ (SD $\pm 560 \mathrm{gm})$, respectively ( $Z>0.05$ for both $G A$ and BW). ${ }^{1}$ Our reported BW is also similar to the previously reported national norm for patients undergoing IVF treatment, which was $3265 \mathrm{gm}(\mathrm{SD} \pm 611)$. However, in our cohort babies were born, on average, 7 days later than that reported by Gibbons et al. ${ }^{5}(38.7 \pm 2.3$ weeks vs. $37.7 \pm 2.2$ weeks, respectively).

\section{Discussion}

In this cohort of women undergoing first fresh cycle of IVF in our center, gestational age at delivery and birth weight did not vary according to infertility diagnoses. We did not find an increased risk for preterm delivery or small for gestational age in our patients. Our results were not different from reported national averages from the general population. ${ }^{1}$ When comparing our results to those reported by Gibbons et al. ${ }^{5}$ in a large cohort of patients in the national SART database, birth weight was not different. However, women in our cohort had an average gestational age at delivery that was seven days longer than results reported in this article. ${ }^{5}$ This finding suggests that some aspect of our dataset is fundamentally different from the national ART data. We were unable to find any clear associations to account for these differences. 
One possible explanation for the longer gestational period in our cohort is the difference in practice patterns between our institution and the other clinics. Specifically, we have an aggressive mandatory single embryo transfer (SET) policy that would have limited the number of women who began with a multifetal gestation. In fact, $46.3 \%$ of women in our cohort underwent SET, and as a result, only $9.32 \%$ of women were documented to have a vanishing twin pregnancy. Our inclusion criteria of singleton delivery has perhaps selected for a larger proportion of patients who underwent SET, however our institution has been reported in the literature to have an overall rate of SET of $28 \%$ for all patients undergoing embryo transfer from June 2004 to May 2009. ${ }^{7}$ This is significantly greater than the national average reported in a survey of the SART database showing a rate of only 4-5\% SET between the years 2004 and $2006 .{ }^{8}$ The presence of a second gestational sac that spontaneously disappears after eight weeks gestation has been associated with poorer obstetrical outcomes in several previous studies, including preterm delivery, low birth weight, very low birth weight, and postnatal mortality in survivor singleton pregnancies $^{9,10}$ Further subgroup analysis within our cohort was considered, but was not performed because of the very small number of women with a vanishing twin.

Another possible explanation for the difference in our results is the limited racial diversity among the women in our cohort. We cannot specifically assess the influence of race on GA and BW in this cohort due to missing data; however, it can be assumed that our population may be somewhat more homogeneous than what is reported in other cohorts. The state census from 2011 reported the lowa population to be $88.4 \%$ white non-Hispanic, $5.2 \%$ Hispanic or Latino, and $3.1 \%$ black. This differs from the nationally reported distribution in the same year of $63.4 \%$ white non-Hispanic, $16.7 \%$ Hispanic or Latino, and $13.1 \%$ black. $^{11}$

Irrespective of the etiology of the difference found in GA for our cohort when compared to the national SART data, it is most important to consider the clinical significance of such a difference. The importance of an additional week of intrauterine growth and development should not be underestimated. Several studies in the pediatric and neonatology literature have suggested that a difference of one week, even at term, can decrease neonatal morbidity and mortality. In one large study from the UK, infants born between 37-38 weeks were at 120 times more likely to require ventilator support for respiratory distress syndrome secondary to surfactant deficiency than were those born at 39 weeks. $^{12}$ 
Table 1. Patient demographics by infertility diagnosis.

\begin{tabular}{|c|c|c|c|c|c|c|c|}
\hline & Cohort & $\begin{array}{l}\text { Male } \\
\text { Factor }\end{array}$ & $\begin{array}{l}\text { Ovulatory } \\
\text { Dysfunction }\end{array}$ & Endometriosis & $\begin{array}{l}\text { Tubal } \\
\text { Factor }\end{array}$ & Unexplained & Combined \\
\hline & $\mathrm{N}=397$ & $\mathrm{~N}=103$ & $\mathrm{~N}=49$ & $\mathrm{~N}=32$ & $\mathrm{~N}=61$ & $\mathrm{~N}=77$ & $\mathrm{~N}=75$ \\
\hline Maternal age $(y)$, mean \pm SD & $31.7 \pm 4.3$ & $30.3 \pm 3.8$ & $31.0 \pm 4.9$ & $33.2 \pm 3.5$ & $32.8 \pm 4.2$ & $32.6 \pm 4.5$ & $32.0 \pm 4.0$ \\
\hline range* & $(21.0-42.0)$ & & & & & & \\
\hline BMI (kg/m2), mean \pm SD & $27.3 \pm 2.4$ & $25.4 \pm 5.5$ & $31.4 \pm 8.1$ & $26.2 \pm 4.8$ & $28.2 \pm 5.9$ & $25.0 \pm 5.3$ & $27.4 \pm 6.7$ \\
\hline range* & $(17.1-51.4)$ & & & & & & \\
\hline \multicolumn{8}{|l|}{ Parity (\%) } \\
\hline $\mathbf{0}$ & 74.8 & 87.4 & 75.5 & 78.1 & 52.5 & 70.1 & 78.7 \\
\hline$\geq 1$ & 25.2 & 12.6 & 24.5 & 21.9 & 47.5 & 29.9 & 21.3 \\
\hline \multicolumn{8}{|l|}{ Day of embryo transfer(5) } \\
\hline Day 3 & 37.8 & 34.0 & 32.7 & 34.4 & 44.3 & 37.7 & 42.7 \\
\hline Day 5 & 62.2 & 66.0 & 67.3 & 65.6 & 55.7 & 62.3 & 64.0 \\
\hline \multicolumn{8}{|l|}{ Mode of delivery (\%) } \\
\hline Vaginal & 64.0 & 70.9 & 53.1 & 62.5 & 62.3 & 63.6 & 64.0 \\
\hline Cesarean section & 36.0 & 29.1 & 46.9 & 37.5 & 37.7 & 36.4 & 36.0 \\
\hline
\end{tabular}

$*$ p value $<0.05$ 
Among infants born by elective cesarean sections, Halliday et al. ${ }^{13}$ found that the risk of neonatal respiratory morbidity decreased with increasing gestational age at delivery, from $7.4 \%$ at 37 weeks to $4.2 \%$ at 38 weeks and $1.8 \%$ at 39 weeks gestation. Highlighting this clinical importance, the American College of Obstetrics and Gynecology recently launched an educational initiative to decrease the rate of elective delivery prior to 39 weeks. $^{14}$

Few groups have examined the association between infertility diagnosis and pregnancy outcome following ART. The results of these studies are incongruous. One study suggests that female infertility diagnosis but not male factor, is associated with reduction in $\mathrm{BW}$ and $\mathrm{GA}^{5}$, whereas another suggests that there is an increased incidence of preterm birth in women with endometriosis and anovulation, but that postnatal admission to the neonatal intensive care unit is increased only in patients with male factor infertility. ${ }^{6}$ Although the findings are not consistent, they suggest that the pathology of the infertility may have a greater influence on birth weight and the length of gestation than has been previously appreciated. Our findings, however, did not support this idea.

Table 2. Mean birth weight and gestational age at delivery by infertility diagnosis*

\begin{tabular}{llll}
\hline Diagnosis & N & $\begin{array}{l}\text { Birth Weight (gms) } \\
\text { (Mean } \pm \text { SD)** }\end{array}$ & $\begin{array}{l}\text { Gestational Age at Delivery (wks) } \\
\text { (Mean } \pm \text { SD)\# }\end{array}$ \\
\hline Male factor & 103 & $3247.3 \pm 129.9$ & $38.7 \pm 0.5$ \\
Ovulatory dysfunction & 49 & $3329.5 \pm 186.4$ & $38.6 \pm 0.7$ \\
Endometriosis & 32 & $3308.8 \pm 228.3$ & $38.4 \pm 0.9$ \\
Tubal factor & 61 & $3424.5 \pm 170.2$ & $38.9 \pm 0.6$ \\
Unexplained & 77 & $3277.9 \pm 147.5$ & $38.9 \pm 0.6$ \\
Combined & 75 & $3228 \pm 149.8$ & $38.2 \pm 0.6$ \\
TOTAL & 397 & $3301.5 \pm 633.8$ & $38.7 \pm 2.3$ \\
\hline
\end{tabular}

*Separate linear regression model adjusted for maternal age, BMI, and parity for each outcome: birth weight and gestational age at delivery

$* * \mathrm{p}=0.61$

$\# \mathrm{p}=0.55$

A recent database study from Finland compared pregnancy outcomes of women who conceived with ART $(n=428)$ with women who were considered subfertile (time to pregnancy of greater than two years) ( $n=928)$. They found no difference in risk of preterm birth or small for gestational age between the groups. Both groups, however, when compared with a control group of over 18,000 pregnancies conceived spontaneously within 6 months, were noted to be at increased risk of preterm birth and low birth weight with odds ratios of 1.56 and 1.92, respectively. This study supports the 
premise that intrinsic maternal factors, perhaps infertility diagnosis itself, and not treatment with ART may play an important role in the risk of preterm delivery and low birth weight pregnancies. ${ }^{15}$ Despite the fact that we expected to find a similar association, our data seems to suggest that rather there is no correlation between infertility diagnosis and obstetrical outcome.

\section{Conclusions}

A strength of this study is the large number of women from a single ART center. Because the number of providers is limited and because our cycles are highly protocol driven, there is less variation in diagnostic coding and practice patterns within our cohort. In addition, all of the embryos were cultured in a single laboratory limiting possible differences in technique. For this reason our outcomes may be less biased than those from a large multicenter analysis. Limitations of this study include the retrospective design and incomplete data points, specifically for smoking status, which is generally thought to have a significant impact on obstetric outcome. It is also important to point out that our population has an unknown proportion of patients using IVF versus IVF with intracytoplasmic sperm injection (ICSI), the latter being associated with a small increased risk for preterm delivery. ${ }^{16}$

In conclusion, the average gestational age at delivery and average birth weight in infants conceived with ART in our cohort did not vary according to maternal infertility diagnosis. When comparing our data to the reported norms from the national SART database, there was no difference in average birth weight, however, our cohort maintained pregnancy for one week longer. This may be related to the aggressive single embryo transfer policy at our institution. Additional studies are needed to further explore the possible impact of SET on gestational age at delivery.

\section{References}

1. Centers for Disease Control and Prevention. 2009 Assisted Reproductive Technology Report. Available at: External link http://www.cdc.gov/art/ART2009/. Accessed October 3, 2011.

2. McDonald SD, Han Z, Mulla S, Murphy $\mathrm{KE}$, Beyene J, Ohlsson A; Knowledge Synthesis Group. Preterm birth and low birth weight among in vitro fertilization singletons: a systematic review and meta-analyses. Eur J Obstet Gynecol Reprod Biol. 2009 Oct;146(2):138-48. doi: 10.1016/j.ejogrb.2009.05.035. Epub 2009 Jul 4. PubMed PMID: 19577836. http://dx.doi.org/10.1016/j.ejogrb.2009.0 5.035

3. Jackson RA, Gibson KA, Wu YW, Croughan MS. Perinatal outcomes in singletons following in vitro fertilization: a meta-analysis. Obstet Gynecol. 2004 Mar;103(3):551-63. PubMed PMID: 14990421.

http://dx.doi.org/10.1097/01.AOG.00001 14989.84822.51

4. Henningsen AK, Pinborg A, Lidegaard $\varnothing$, Vestergaard C, Forman JL, Andersen AN. Perinatal outcome of singleton siblings born after assisted reproductive technology and spontaneous conception: Danish national siblingcohort study. Fertil Steril. 2011 Mar 1;95(3):959-63. doi: 10.1016/j.fertnstert.2010.07.1075. PubMed PMID: 20813359. 
5. Gibbons WE, Cedars M, Ness RB; Society for Assisted Reproductive Technologies Writing Group. Toward understanding obstetrical outcome in advanced assisted reproduction: varying sperm, oocyte, and uterine source and diagnosis. Fertil Steril. 2011 Apr;95(5):1645-9.e1. doi: 10.1016/j.fertnstert.2010.11.029. Epub 2010 Dec 3. PubMed PMID: 21130432.

6. Kuivasaari-Pirinen $\mathrm{P}$, Raatikainen $\mathrm{K}$, Hippelainen $M$, Heinonen S. Adverse outcomes of IVF/ICSI pregnancies depending on aetiology of infertility. ISRN Obstetrics and Gynecology, Volume 2012 (2012), Article 451915, 5 pages. DOI: 10.5402/2012/451915.

7. Kresowik JD, Stegmann BJ, Sparks AE, Ryan GL, van Voorhis BJ. Five-years of a mandatory single-embryo transfer (mSET) policy dramatically reduces twinning ratewithout lowering pregnancy rates. Fertil Steril. 2011 Dec;96(6):13679. doi:10.1016/j.fertnstert.2011.09.007. Epub 2011 Sep 29. PubMed PMID: 21962964.

8. Luke $B$, Brown MB, Grainger DA, Cedars M, Klein N, Stern JE; Society for Assisted Reproductive Technology Writing Group. Practice patterns and outcomes with the use of single embryo transfer in the United States. Fertil Steril. 2010 Feb;93(2):490-8. doi: 10.1016/j.fertnstert.2009.02.077. Epub 2009 Apr 18. PubMed PMID: 19376512.

9. Pinborg $A$, Lidegaard $O$, la Cour Freiesleben $\mathrm{N}$, Andersen AN. Consequences of vanishing twins in IVF/ICSI pregnancies. Hum Reprod. 2005 Oct;20(10):2821-9. Epub 2005 Jun 24. Erratum in: Hum Reprod. 2006 May;21(5):1335. PubMed PMID: 15979998.

http://dx.doi.org/10.1093/humrep/dei142
10. Sazonova A, Källen K, Thurin-Kjellberg A, Wennerholm UB, Bergh C. Factors affecting obstetric outcome of singletons born after IVF. Hum Reprod. 2011 Oct;26(10):2878-86. doi: 10.1093/humrep/der241. Epub 2011 Jul 19. PubMed PMID: 21771774.

11. United States Census Bureau. State and County Quick Facts. Available at: External link http://quickfacts.census.gov/qfd/states/1 9000.html . Accessed July 18, 2012.

12. Madar J, Richmond S, Hey E. Surfactant-deficient respiratory distress after elective delivery at 'term'. Acta Paediatr. 1999 Nov;88(11):1244-8. PubMed PMID: 10591427. http://dx.doi.org/10.1080/080352599750 030365

13. Halliday J. Outcomes of IVF conceptions: are they different? Best Pract Res Clin Obstet Gynaecol. 2007 Feb;21(1):67-81. Epub 2006 Oct 19. PubMed PMID: 17055783. http://dx.doi.org/10.1016/j.bpobgyn.2006 .08 .004

14. The American College of Obstetricians and Gynecologists. Elimination of NonMedically Indicated (Elective) Deliveries before 39 Weeks Gestational Age. Available at: External link http://www.acog.org/About_ACOG/ACO G_Districts/District_II/Less_Than_39_W eeks_Deliveries. Áccessed July 18 , 2012.

15. Raatikainen $K$, Kuivasaari-Pirinen $P$, Hippeläinen $M$, Heinonen $S$. Comparison of the pregnancy outcomes of subfertile women after infertility treatment and in naturally conceived pregnancies. Hum Reprod. 2012 Apr;27(4):1162-9. doi:10.1093/humrep/des015. Epub 2012 Feb 14. PubMed PMID: 22333986. 
16. Alukal JP, Lamb DJ. Intracytoplasmic sperm injection (ICSI)--what are the risks? Urol Clin North Am. 2008 May;35(2):277-88, ix-x. doi:10.1016/j.ucl.2008.01.004. Review. PubMed PMID: 18423248; PubMed Central PMCID: PMC2424218. 